\title{
Why the universe is unexpectedly transparent to very high energy gamma-rays
}

\author{
Hassan Abdalla * \\ Centre for Space Research, North-West University, Potchefstroom 2520, South Africa \\ Department of Astronomy and Meteorology, Omdurman Islamic University, Omdurman 382, \\ Sudan \\ E-mail: hassanahh@gmail.com
}

\section{Markus Böttcher}

Centre for Space Research, North-West University, Potchefstroom 2520, South Africa

E-mail: Markus.Bottcheranwu.ac.za

\begin{abstract}
Recent observations of distant gamma ray sources indicate that the universe may be more transparent to VHE-gamma-rays than expected. In this paper we study the reduction of the EBL gamma-gamma opacity due to the existence of underdense regions along the line of sight to VHE gamma-ray sources and the possibility of a Lorentz Invariance Violation (LIV) signature. Therefore, we study whether one or both of these effects could be suitable to explain the spectral hardening observed in a few VHE gamma-ray sources. We found that, although the cosmic opacity for VHE gamma rays with energy more than $10 \mathrm{TeV}$ can be strongly reduced, the spectral hardening feature observed in some VHE gamma-ray blazars with energy from $300 \mathrm{GeV}$ up to few TeVs (e.g. PKS 1424+240) still remains puzzling.
\end{abstract}

5th Annual Conference on High Energy Astrophysics in Southern Africa

4-6 October, 2017

University of the Witwatersrand (Wits), South Africa

${ }^{*}$ Speaker. 


\section{Introduction}

The extragalactic Very High Energetic (VHE) photons from distant gamma-ray sources (e.g. blazars) can be absorbed due to $\gamma \gamma$ absorption by low-energy extragalactic background light (EBL) photons. The photon flux attenuation due to this process and its importance in high-energy astrophysics was first pointed out by Nishikov in 1962 [1], which has shown that the probability of conversion of $\mathrm{TeV}$ gamma ray traversing a length comparable to the Hubble distance into an electron-positron pair due to it is interaction with soft EBL photons, is appreciable. Currently, the study of the opacity or transparency of the Universe to VHE photons coming from high redshift astronomical objects due to their interaction with intergalactic EBL photons is a very active area of high-energy astrophysics, because of its potential to study the cluster environments of blazars [2] and estimate some cosmological parameters [3]. However, the EBL is very difficult to measure directly because of the strong foreground emissions within our galaxy and solar system (e.g. atmosphere and zodiacal light ) [4].

Several important studies have been carried out to estimate the average EBL energy density and its cosmological evolution, and to calculate the predicted $\gamma \gamma$ absorption imprints, by employing a variety of theoretical and empirical research methods $[5,6,7,8,9,10,11,12,13]$.

Recent observations of some distant VHE-gamma rays sources (e.g. blazars) seem to indicate hard VHE gamma-ray spectra which have been interpreted by several authors as a hint that the Universe might be more transparent to VHE gamma-rays than expected from current EBL models. $[14,15,16]$. There have been many attempts to find possible explanations for this VHE-gamma ray signature in some distant blazar spectra. Possible solutions include the hypothesis that the EBL density is generally lower than expected from current models [17]; the existence of exotic Axion Like Particles (ALPs) [14, 18]; photo-pion-production interactions of extragalactic Ultra-high-Energy Cosmic Rays (UHECRs) along the line of sight [19]; and EBL inhomogeneities [20, 21, 22].

In a previous paper [22] we investigated the effects of inhomogeneities of the EBL on the gammagamma opacity using the prescription of Razzaque et al. (2014) [9].

In this paper, to include the additional EBL component from mid- to far infrared, by re-processing of starlight, we used the model of Finke et al. (2015) [10]. To calculate the resulting $\gamma \gamma$ opacity for VHE $\gamma$-ray photons from sources at cosmological distances, due to their interaction with intergalactic radiation fields (EBL) through electron-positron pair production, we assumed a VHE-gamma ray source located at redshift 0.6 . Then, we investigate the effect of cosmic voids along the line of sight to the VHE- $\gamma$-rays source, and the effect of LIV, to compare the two effects, and discuss in which energy band each is dominant. 


\section{Cosmic Voids Along the Line of Sight}

To study the impact of an under-dense region, a generic study of the effects of cosmic voids along the line of sight to a blazar has been done [22], in which we assumed that a spherical cosmic void with radius $R$ is located with its center at redshift $z_{v}$, between the observer and the VHE $\gamma$-ray source located at redshift $z_{s}$. We calculate the angle- and photon-energy-dependent EBL energy density at each point between the observer at redshift zero and the source by using co-moving cordinates, converting redshifts $z$ to distances $l(z)$. The cosmic void is represented by setting the star formation rate to 0 within the volume of the void. Following Razzaque et al. (2014), only the direct starlight contribution to the EBL was considered in [22]. From figure (1), we notice that the

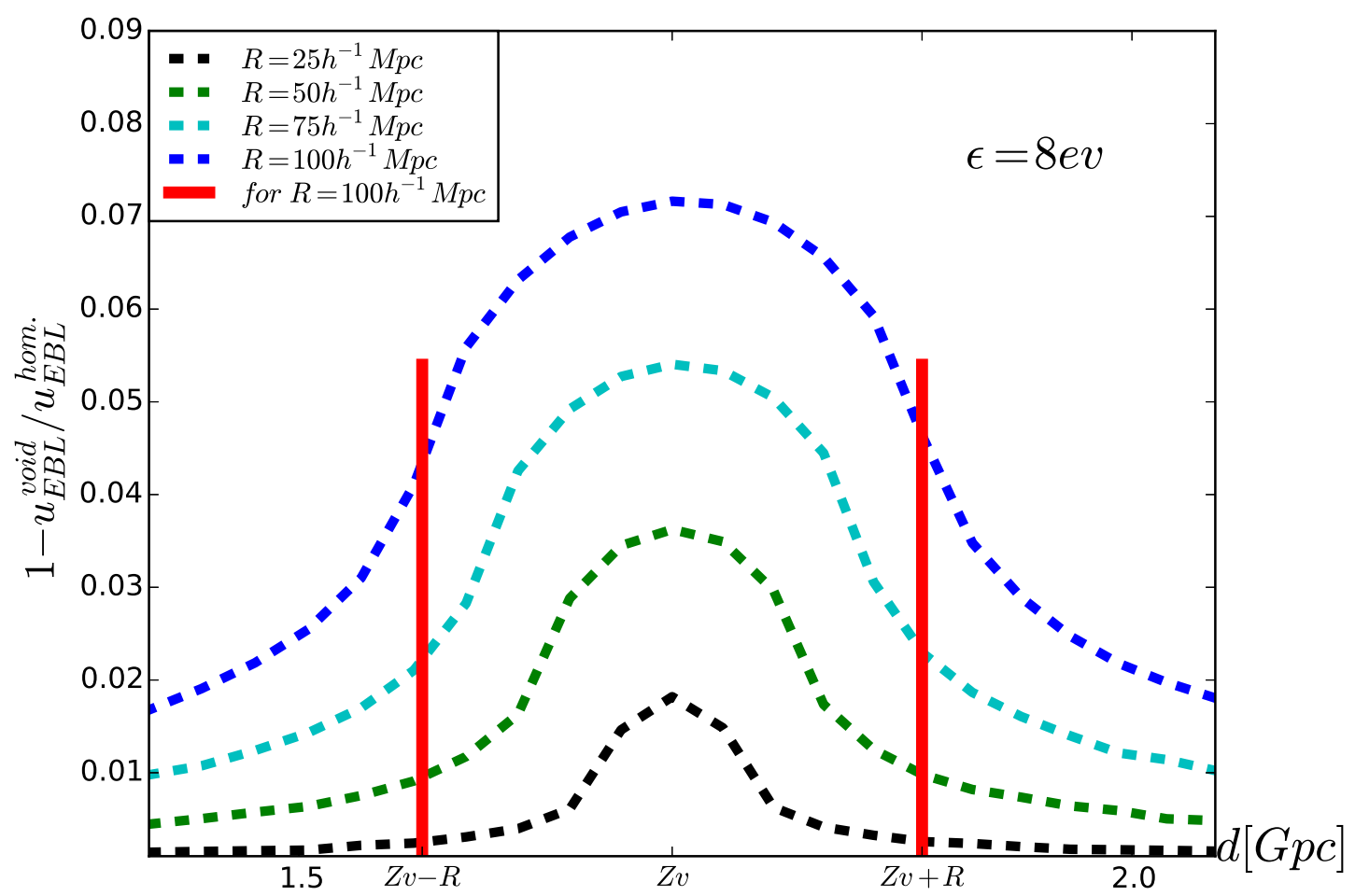

Figure 1: The relative EBL photon energy density deficit due to the presence of a void of various sizes (as indicated by the labels) as a function of distance along the line of sight of a void located at $z_{v}=0.5$, at a representative photon energy of $\varepsilon=8 \mathrm{eV}$. The red vertical lines indicate the boundaries of the void for the $R=100 h^{-1}$ Mpc case [22].

EBL deficit is proportional to the size of the void. Therefore, the effect of a number $n$ of voids of radius $R_{1}$ is aproximately the same as the effect of a large void with radius $R_{n}=n R_{1}$. Detailed calculations, which assume voids along the line of sight using the Razzaque. et al. (2009) [9] EBL model, can be found in [22].

In this work we calculate the impact of cosmic voids along our line of sight to a distant VHE gamma-ray source, by using the model developed by by Finke, et al. (2015) [10]. In this model, 
the full EBL spectrum (from far infrared through visible and extending into the ultraviolet) is calculated, by considering the stars that evolved off the main sequence and re-emission of absorbed starlight by dust. More details can be found in [10]. We investigate the impact of the existence of an accumulation of about 10 voids of typical sizes with radius $R=100 \mathrm{~h}^{-1} \mathrm{Mpc}$ centered at redshift $z_{v}=0.3$ along the line of sight to an object (e.g. ablazar) located at redshift $z_{v}=0.6$, which is presented in figure (2).

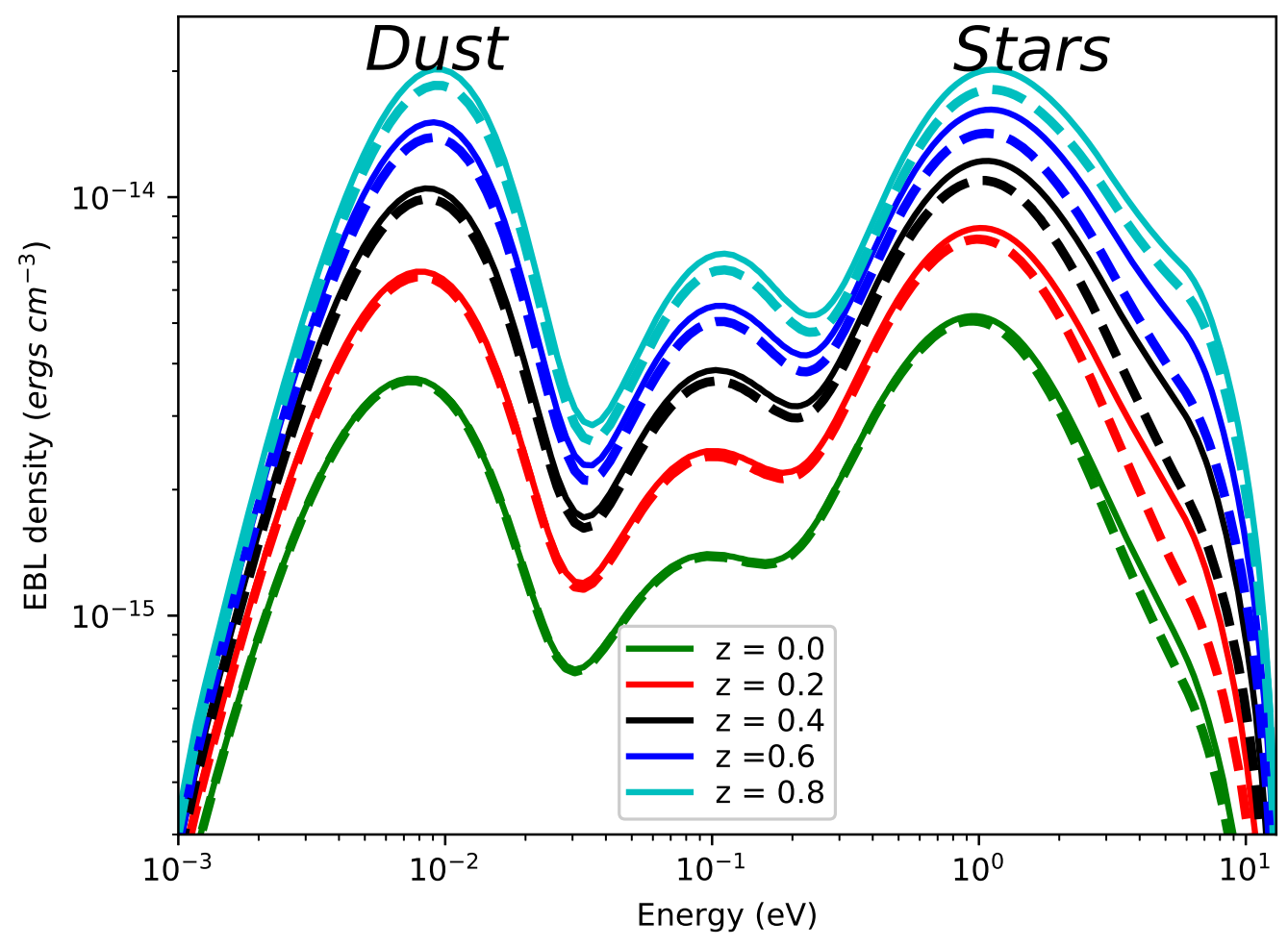

Figure 2: Differential EBL photon energy density as a function of distance along the line of sight. The solid lines $(R=0)$ represent the homogeneous case, and the dashed lines represent the EBL energy density considering an accumulation of about 10 voids of typical sizes with radius $R=100 \mathrm{~h}^{-1} \mathrm{Mpc}$ centered at redshift $z_{v}=0.3$. The general increase of the EBL energy density with redshift is due to the increasing star formation rate with redshift. 
In figure (2) we present the EBL energy density spectrum (the full spectrum from 0.001 to $10 \mathrm{eV}$ including the direct emission, and absorption and reradiation by dust) for the case of a void (dashed lines), compared to the homogeneous case (solid lines) at different points (redshifts, as indicated by the labels) along the line of sight. To represent the difference between the homogeneous

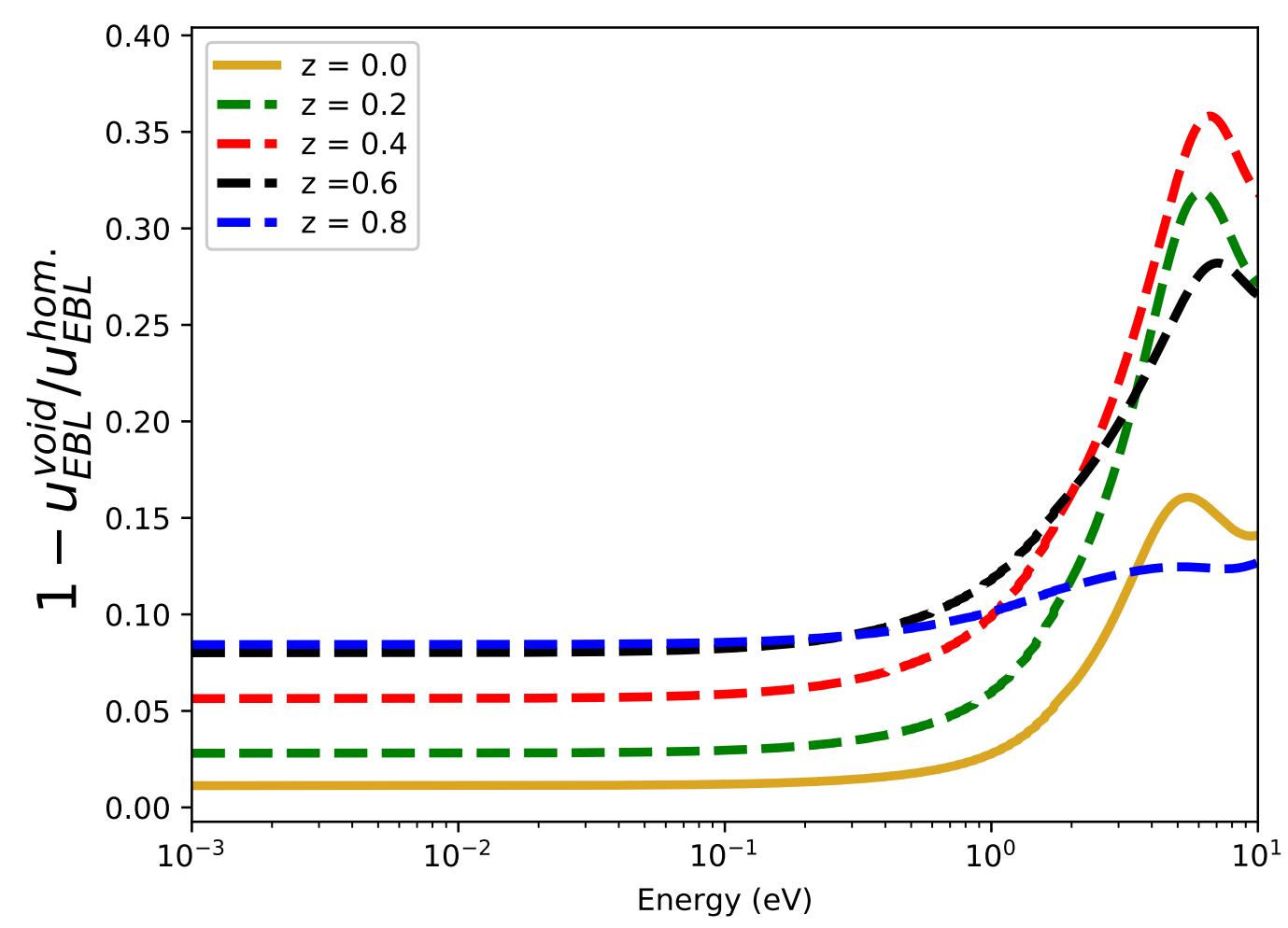

Figure 3: Relativie EBL energy density deficit due to the presence of a void for the same cases as represented in figure (2). The fraction between the homogeneous and inhomogeneous cases for different redshifts presented in this figure.

and inhomogeneous cases clearly, we calculated the fractional difference between the homogeneous and the inhomogeneous case as a function of photon energy for various redshifts along the line of sight. The result is plotted in figure (3). Figure (3) illustrates that the EBL deficit is smaller for low-energy (IR) photons than for optical - ultraviolet photons. This is because the UV EBL is dominated by hot, young stars, thus more strongly reflecting the local effect of the void.

Since in this work we set only the star formation rate inside the void equal to zero, some dust reprocessing still takes place inside the void.

From figure (3), we can notice that the relative EBL energy density deficit due to the presence of voids is quite significant only at (and around) the center of the voids but the deficit close to the edge of the void or just outside of the void is very small. Therefore the reduction of the gamma-gamma opacity due to the cosmic voids is expected to be insignificant; the optical depth deficit for the VHE-gamma rays due to the possibility of cosmic voids is investigated in Sections (3) and (4). 


\section{Lorentz Invariance Violation}

Lorentz invariance (or Lorentz symmetry) is one of the fundamental concepts in the Special Theory of Relativity. But recently, several quantum-gravity theories predict that familiar concepts such as Lorentz symmetry can be broken at energies approaching the Planck energy scale $\left(E_{L I V} \sim 1.2 \times 10^{19} \mathrm{GeV}\right)$. While such extreme energies are currently unreachable by experiments, tiny residual effects at attainable energies can be measured when VHE photos propagate over sufficiently large distances. Thus, for photons traveling over cosmological distances, the accumulated deviations from the Lorentz symmetry might be large enough to be measured. The deviation from Lorentz symmetry can be described by a modification of the dispersion relation as follows $[23,24,25,26,27]$ :

$$
E^{2}=p^{2} c^{2}+m^{2} c^{4} \pm E^{2}\left(\frac{E}{\zeta_{n} E_{L I V}}\right)^{n}
$$

where $c$ is the conventional speed of light, the dimensionless parameter $\zeta_{n}$ and the order of the leading correction $n$ are depending on particle type and theoretical framework. The values $n=1,2$ are the cases most often considered. The" - sign" represents a subluminal scenario (decreasing photon speed with increasing energy), and the "+ sign" represents the superluminal case (increasing photon speed with increasing energy) $[23,28]$. The violation of Lorentz invariance leads to a modification of the threshold energy and cross section of the gamma-gamma pair production process. This can lead to an energy-dependent refractive index for light in vaccum, with higher energy photons propagating more slowly than their lower-energy counterparts [28]. The modified pair-production threshold energy $\varepsilon_{\min }$, can be obtained solely on the minimal assumption that the standard energy-momentum conservation still holds in the Lorentz violation framework (Note: this may not be true in some LIV schemes). To lowest non-trivial order in $E / E_{L I V}$, the modified pair-production threshold energy $\varepsilon_{\min }$ by considering the subluminal case only, can be written as [29]:

$$
\varepsilon_{\min }=\frac{m^{2} c^{4}}{E_{\gamma}}+\left(\frac{E^{2}}{4 E_{L I V}}\right)
$$

where $E$ is the considered VHE-photon energy and $E_{L I V}$ is the relevant energy scale, which is commonly assumed to be of the order of the Planck energy. For energies $E \ll E_{L I V}$, any deviation from Lorentz symmetry is expected to be very small. The resulting threshold energy $\varepsilon_{\min }$ of a target photon as a function of its VHE gamma-ray energy is shown in figure (4). In this figure we represented the standard quantum electrodynamics case by a black solid line and the dashedlines indicate the modified threshold energy for different $E_{L I V}$ values (in units of $10^{19} \mathrm{GeV}$ ). The standard relation for optical depth at the energy $E_{\gamma}$ and for a source at redshift $z_{s}$ is modified as [29]:

$$
\tau_{\gamma \gamma}\left(E_{\gamma}, z_{s}\right)=\frac{c}{8 E_{\gamma}^{2}} \int_{0}^{z_{s}} \frac{d z}{H(z)(1+z)^{3}} \int_{\mathcal{E}_{\min }}^{\infty} \frac{n(\varepsilon, z)}{\varepsilon^{2}} \int_{s_{\min }}^{s_{\max (z)}}\left(s-\frac{E_{\gamma}^{3}}{E_{L I V}}\right) \sigma_{\gamma \gamma}(s) d s,
$$

where $H(z)=H_{0} \sqrt{\left[\Omega_{m}(1+z)^{3}+\Omega_{\Lambda}\right]}, n(\varepsilon, z)$ is the EBL photon number density as a fuction of redshift $z$ and energy $\varepsilon$, and $\sigma_{\gamma \gamma}(s)$ is the total pair production cross section as a function of the modified square of the center of mass energy $s=-\frac{E^{3}}{E_{L I V}}+2 \varepsilon E_{\gamma}(1-\cos (\theta))$, where $\theta$ is the angle between the soft EBL photon of energy $\varepsilon$ and the VHE gamma ray photon. The limits of the last 


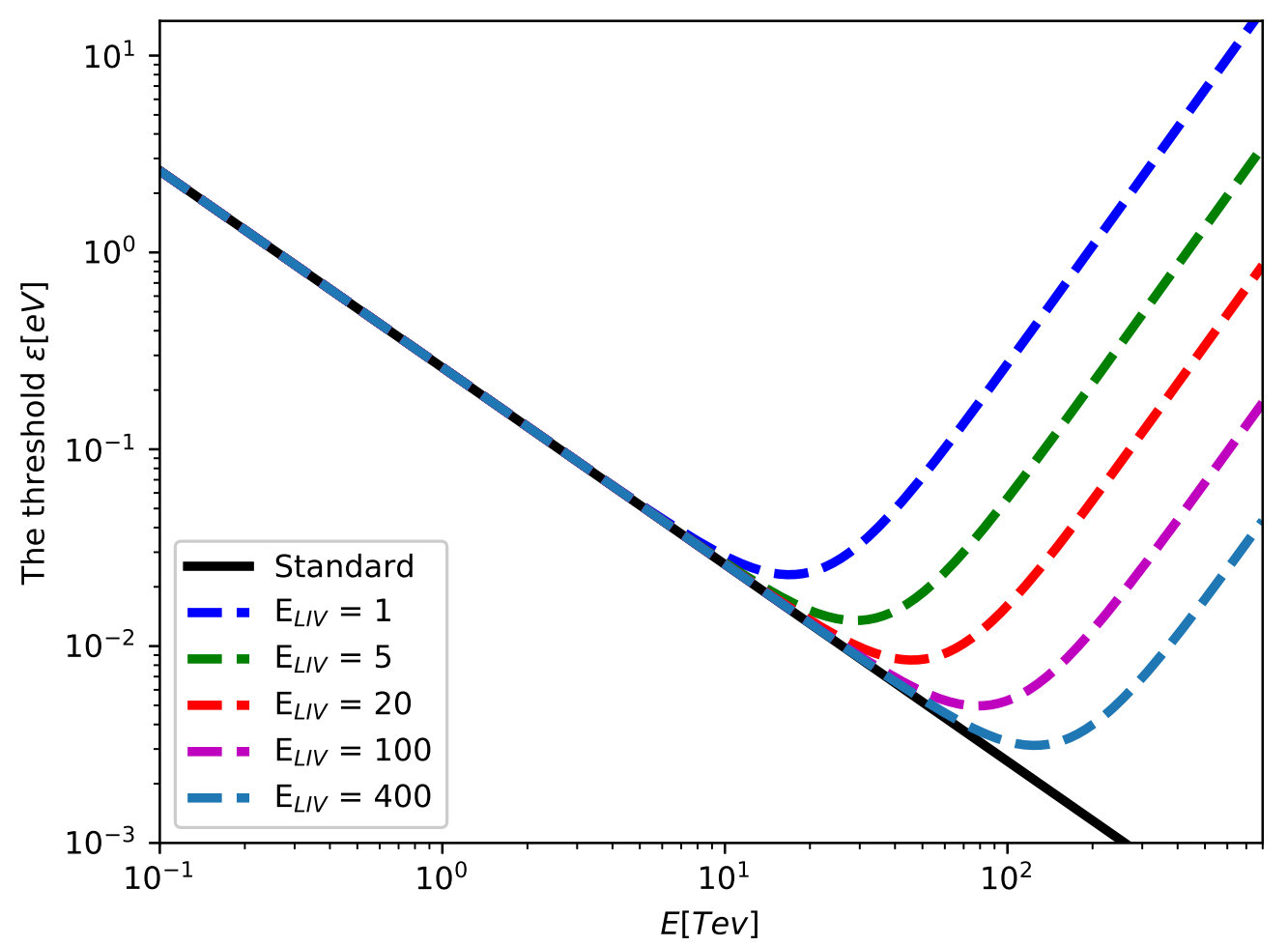

Figure 4: This figure (reproduced from [29]), gamma-gamma pair production threshold energy as a function of VHE gamma-ray photon energy (Equation (3.2)). The black solid-line shows the standard case and the dashed lines indicate the modified threshold due to the effect of LIV, for different values of the parameter $E_{L I V}$ (in the units of $10^{19} \mathrm{GeV}$ ).

integral can be written as $s_{\min }=4 m_{e}^{2} c^{4}$ and $s_{\max }=4 \varepsilon E_{\gamma}(1+z)-\frac{E_{\gamma}^{3}}{E_{L I V}}$. Note that the kinematics outlined above take into account the modified dispersion relation only for photons, but not for electrons. From equation 3.3, it is obvious that when $E_{L I V} \longrightarrow \infty$, the standard relations are recovered. In the following, it is assumed that the expression for the cross section as a function of $s$ remains unchanged.

\section{Cosmic Voids and LIV}

We compare the effects of inhomogeneities due to the presence of cosmic voids to those of LIV in figure (5). For comparison purposes, to study the effect of cosmic voids along the line of sight and/or of LIV, in figure (6) we plot the relativie optical depth $\tau_{\gamma \gamma}$ deficit as a function of energy for VHE-gamma rays that propagate from a source located at redshift $z=0.6$. The effect of a cosmic void along the line of sight is presented by the black dot-dashed line. As already elaborated in [22], the effect of a plausible EBL inhomogeneity on the gamma-gamma opacity is not sufficient to explain the unexpected spectral hardening of, e.g., the blazar PKS 1424+240. We also notice that the optical depth $\tau_{\gamma \gamma}$ deficit decreases with energy and is very small for VHE 


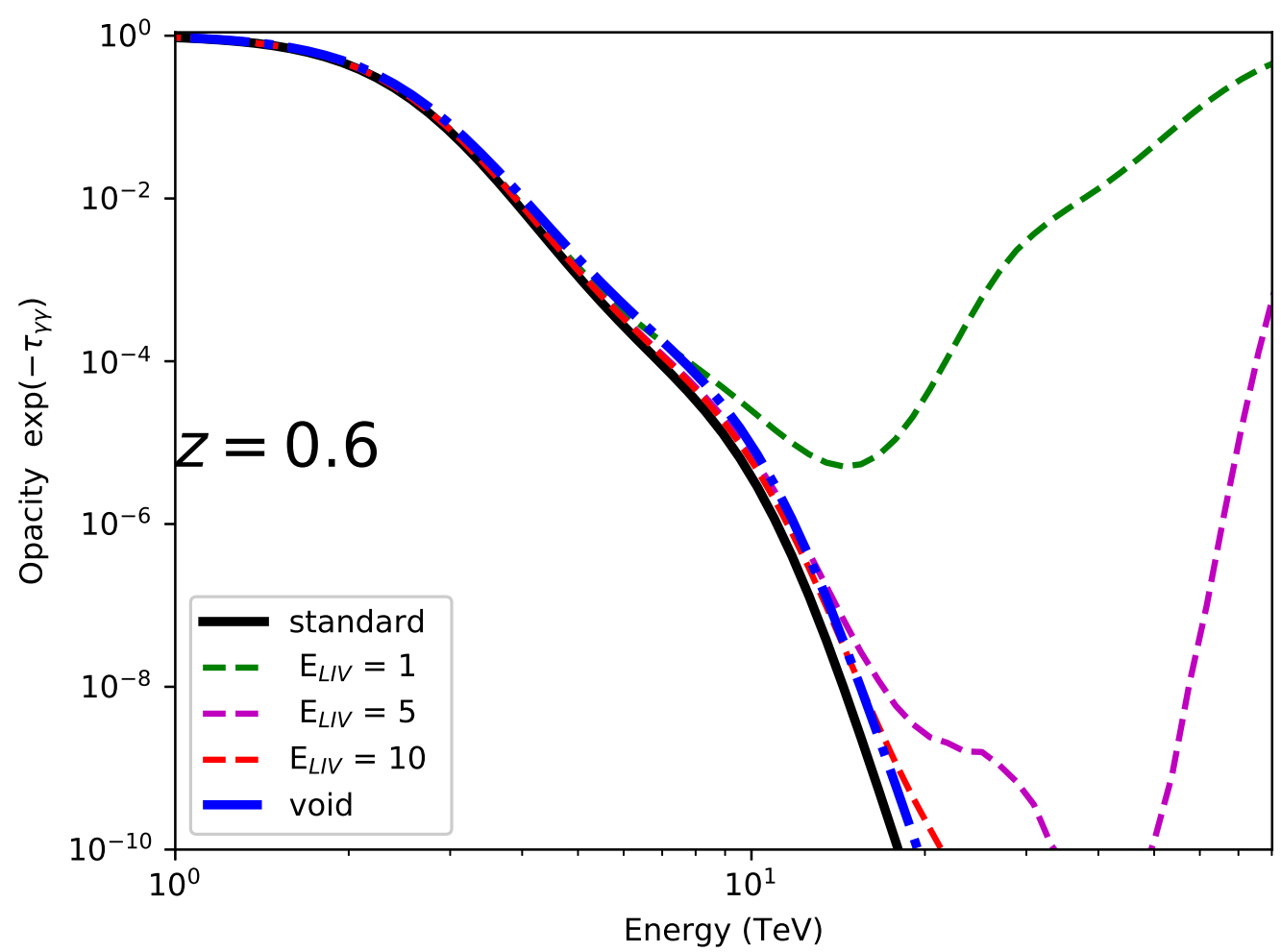

Figure 5: Absorption coefficient $\exp \left(-\tau_{\gamma \gamma}\right)$ as a function of energy for VHE-gamma rays that propagate from a source located at redshift $z=0.6$, using the EBL model devoloped by Finke, et al. (2015) [10]. The black solid line represents the case of standard quantum electrodynamics (QED) physics and using the homogeneous EBL energy density distribution, and the blue dot-dashed line represents the case of standard QED and EBL energy density calculated by considering an accumulation of 10 voids of typical sizes with radius $R=100 h^{-1} \mathrm{Mpc}$ centered at redshift $z_{v}=0.3$. The dashed-lines show the modified coefficient for different values of $E_{L I V}$ in the unit of Planck energy scale $\left(1.2 \times 10^{19} \mathrm{GeV}\right)$.

gamma-ray photons above $\sim 10 \mathrm{TeV}$. To study the effects of LIV, we again use the EBL model of Finke, et al. (2015) [10], and calculate both the effect of LIV alone, as well as the combined effect of LIV and a cosmic void. In figure (6), the green, blue and red lines represent the deficit due to the LIV modification, for different values of $E_{L I V}$ and the violet dashed line due to both effects. We can notice that the LIV effect is very small for energies below about $5 \mathrm{TeV}$, but very significant at higher energy $E \approx 10 \mathrm{TeV}$ or higher.

Our results show that, due to LIV, one may expect VHE gamma-ray photons beyond $10 \mathrm{TeV}$ to be observable from cosmological sources, but even the combination of LIV and cosmic voids is insufficient to explain the spectral hardening in the $\mathrm{TeV}$ regime seen in several blazars. 


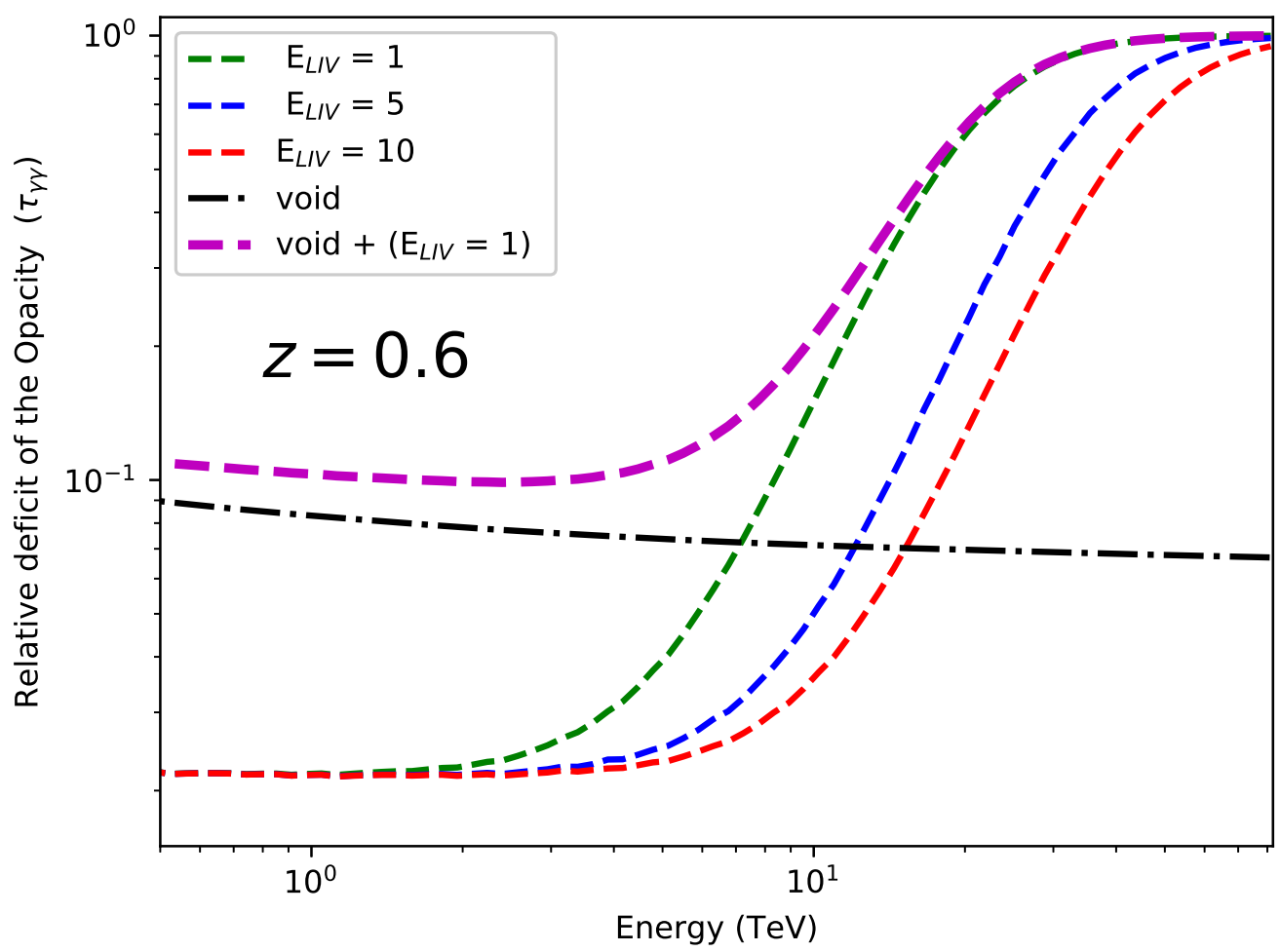

Figure 6: Relativie optical depth $\tau_{\gamma \gamma}$ deficit as a function of energy for VHE-gamma rays that propagate from a source located at redshift $z=0.6$, using Finke, et al. (2015) [10] EBL model. The black-dashed line represent the case that, the deficit due to that the EBL energy density calculated by considering an accumulation of about 10 voids of typical sizes with radius $R=100 h^{-1} \mathrm{Mpc}$ centered at redshift $z_{v}=0.3$. The green, blue, red lines represent that, the deficit due to the correction from LIV for different values of $E_{L I V}$ in the unit of Planck energy scale $\left(1.2 \times 10^{19} \mathrm{GeV}\right)$ and the top dashed line due to the both effects.

\section{Summary and Conclusions}

We have presented calculations of modifications of the gamma-gamma opacity for VHE $\gamma$-ray photons from sources at cosmological distances, due to their interaction with intergalactic radiation fields (EBL) through electron-positron pair production. We evaluated both the effects of cosmic voids along the line of sight and of Lorentz-invariance violating extensions of the standard model. We found that the optical depth deficit for VHE-gamma rays due to the existence of cosmic voids along our line of sight to distant blazars is not enough to explain the unexpected spectral hardening of the VHE spectra of several blazars (e.g. PKS 1424+240).

We found that the optical depth $\tau_{\gamma \gamma}$ deficit due to voids decreases with energy and becomes negligible for VHE gamma-ray photons above $\sim 10 \mathrm{TeV}$. We confirm previous results that the effect of LIV becomes important only at VHE energies above $\sim 10 \mathrm{TeV}$. Even if we consider the combination of both effects (cosmic void along the line of sight and LIV) the reduction of the EBL gamma-gamma opacity is too small to explain the unexpectedly hard VHE gamma-ray spectra of 
some blazars. More detailed calculations considering a subluminal and a superluminal modification of the dispersion relation for photons for the LIV effect, by considering $\gamma \gamma$ absorption and also the impact of LIV effect on the Compton scattering process are presented in [30].

The future Cherenkov Telescope Array with its significant sensitivity above $10 \mathrm{TeV}$ will offer the opportunity to test LIV extensions of the standard model due to the resulting reduction of the EBL opacity at those energies, and thus offer an exciting window on fundamental physics [28]. However, the spectral hardening of several observed VHE gamma-ray sources (e.g. blazars) with energy from $300 \mathrm{GeV}$ up to few TeVs (e.g. PKS 1424+240) still remains puzzling. Therefore, other explanations for such hardening must be invoked. One possibility is that this hardening is, in fact, a real, intrinsic feature of the $\gamma$-ray spectra of these blazars, possibly due to a pion-production induced cascade component in a hadronic blazar model scenario [31, 32]. If such a spectral hardening is not intrinsic to the source, more exotic explanations, such as ALPs or a cosmic-ray induced secondary radiation component, would need to be invoked.

\section{References}

[1] Nikishov, A. I. 1962, Sov. Phys. JETP, 14, 393

[2] Sushch, I. \& Böttcher, M. 2015, A \& A, 573, A47

[3] Biteau A. \& Williams D. A. 2015, ApJ, 812, 1

[4] Hauser, M. G. \& Dwek, E. 2001, ARA\&A, 39, 249

[5] Stecker, F. W. 1969, ApJ, 157, 507

[6] Stecker, F. W., de Jager, O. C., \& Salamon, M. H., 1992, ApJ, 390, L49

[7] Aharonian F. et al. 2006, Nature, 440, 1018

[8] Franceschini, A., Rodigheiro, G., \& Vaccari, M., 2008, A\&A, 487, 837

[9] Razzaque S., Demer, C. D., \& Finke, J. D., 2009, ApJ, 697, 483

[10] Finke, J. D., Razzaque, S., \& Dermer, C. D., 2010, ApJ, 712, 238

[11] Dominguez, A., et a., 2011a, MNRAS, 410, 2556

[12] Gilmore, R. C., Sommerville, R. S., Primack, J. R., \& Dominguez, A., 2012, MNRAS, 422, 3189

[13] H.E.S.S. Collaboration: Abdalla, H., et al. 2017, Astron. Astrophys., 606, A59

[14] Dzhatdoev T.A., et al. 2017, Astron. Astrophys., 603, A59

[15] MAGIC Collaboration: Albert, J., et al., 2008, Science, 320, 1752

[16] Archambault S. et al. 2014, ApJ, 785, L16

[17] Furniss A., et al., 2013, ApJ, 768, L31

[18] Dominguez, A., Sánchez-Conde, M. A., \& Prada, F., 2011b, J. Cosmol. Astropart. Phys., 11, 020

[19] Essey W. \& Kusenko A. 2010, ApJ, 33, 81

[20] Furniss A., Stutter, P. M., Primack, J. R., \& Dominguez, A., 2015, MNRAS, 446, 2267

[21] Kudoda, A. M. \& Faltenbacher, A., 2016, PoS, HEASA2015, 20

[22] Abdalla, H. \& Böttcher, M., 2017, ApJ, 835, 237 
[23] Amelino-Camelia, G., et al. 1998, Nature, 393, 763

[24] Garay, L., J. 1995, Int. J. Mod. Phys. A, 10, 145

[25] Mattingly, D. 2005, Liv. Rev. Relat., 8, 5

[26] Martínez-Huerta, H. \& Pẽrez-Lorenzana, A., 2017, Phys. Rev. D95, 063001

[27] Chrétien, M., Bolmont, J., Jacholkowski, A. 2016, ICRC 2015, 34, 764, arXiv: 1509.03545

[28] Fairbairn M. et al. 2014, JCAP, 06, 005

[29] Tavecchio, F. \& Bonnoli, G., 2016, Astron. Astrophys., 585, A25

[30] Abdalla, H. \& Böttcher, M., 2018, ApJ, 865, 159, arXiv: 1809.00477v1

[31] Böttcher, M., Reimer, A., Sweeney, K., \& Prakash, A., 2013, ApJ, 768, 54

[32] Cerruti, M., Zech, A., Boisson, C., \& Inoue, S., 2015, MNRAS, 448, 910 